\title{
Tunicamycin enhances TRAIL-induced apoptosis by inhibition of cyclin D1 and the subsequent downregulation of survivin
}

\author{
Hai-Yan Zhang ${ }^{1,4}$, Zhen-Xian Du², Bao-Qin Liü, \\ Yan-Yan $\mathrm{Gao}^{3}$, Xin Meng ${ }^{3}$, Yifu Guan ${ }^{3}$, \\ Wei-Wei Deng ${ }^{1}$ and Hua-Qin Wang ${ }^{3}$ \\ ${ }^{1}$ Department of Geriatrics \\ ${ }^{2}$ Department of Endocrinology and Metabolism \\ The First Affiliated Hospital \\ ${ }^{3}$ Department of Biochemistry and Molecular Biology \\ China Medical University \\ Shenyang 110001, China \\ ${ }^{4}$ Corresponding author: Tel, 86-24-81958908; \\ E-mail, zhy_doctor@hotmail.com \\ DOI 10.3858/emm.2009.41.5.041
}

Accepted 4 March 2009

Abbreviations: DcR, decoy receptor; DISC, death-inducing signaling complex; DR, death receptor; TRAIL, TNF-related apoptosis-inducing ligand

\begin{abstract}
TNF-related apoptosis-inducing ligand (TRAIL) has been proposed as a promising cancer therapy that preferentially induces apoptosis in cancer cells, but not most normal tissues. However, many cancers are resistant to TRAIL by mechanisms that are poorly understood. In this study, we showed that tunicamycin, a naturally occurring antibiotic, was a potent enhancer of TRAIL-induced apoptosis through downregulation of survivin. The tunicamycin-mediated sensitization to TRAIL was efficiently reduced by forced expression of survivin, suggesting that the sensitization was mediated at least in part through inhibition of survivin expression. Tunicamycin also repressed expression of cyclin $\mathrm{D} 1$, a cell cycle regulator commonly overexpressed in thyroid carcinoma. Furthermore, silencing cyclin D1 by RNA interference reduced survivin expression and sensitized thyroid cancer cells to TRAIL; in contrast, forced expression of cyclin D1 attenuated tunicamycin-potentiated TRAIL-induced apoptosis via over-riding downregulation of survivin. Collectively, our results demonstrated that tunicamycin promoted TRAIL-induced apoptosis, at least in part, by inhibiting the expression of cyclin D1 and subsequent survivin. Of note, tunicamycin did not sensitize the differentiated thyroid epithelial cells to
\end{abstract}

TRAIL-induced apoptosis. Thus, combined treatment with tunicamycin and TRAIL may offer an attractive strategy for safely treating resistant thyroid cancers.

Keywords: apoptosis; BIRC5 protein, human; cyclin D1; thyroid neoplasms; TNF-related apoptosis-inducing ligand; tunicamycin

\section{Introduction}

Undifferentiated thyroid carcinomas are refractory to most traditional chemotherapy and radiotherapeutic methods (Ain, 1998). TNF-related apoptosis-inducing ligand (TRAIL) initiates apoptosis by binding to its cell surface death receptors 4 and 5 (DR4 and DR5), which contain a cytoplasmic death domain that serves as a protein interaction module to recruit the death domain-containing adaptor protein FADD to the death-inducing signaling complex (DISC) (LeBlanc et al., 2003). The signal is propagated through caspases 8 and 10, finally leading to activation of effector caspases such as caspase 3 (Sprick et al., 2000; Wang et al., 2001). TRAIL also has three decoy receptors (DcR1, DcR2 and osteoprotegerin), which inhibit apoptosis by sequestering TRAIL from DR4 and DR5 death receptors (LeBlanc et al., 2003).

Recent studies showed that TRAIL may be considered as a promising anticancer agent due to its ability to induce apoptosis in a variety of tumor cell types with only negligible effects on normal cells (Pan et al., 1997a, b). However, malignant thyroid cancer cells exhibit considerable heterogeneity in their sensitivity to TRAIL, even among those expressing the TRAIL-responsive cognate death receptors, DR4 and DR5 (Bretz et al., 1999a, b; Mitsiades et al., 2000; Wang et al., 2004). Clearly, the identification of drugs that sensitize tumors to TRAIL-induced apoptosis, without compromising its tumor selectivity, could substantially broaden the therapeutic impact of TRAIL.

Tunicamycin, a naturally occurring antibiotic, blocks the first step in the biosynthesis of $\mathrm{N}$-linked oligosaccharides in cells. Our present experiments demonstrate that tunicamycin significantly increases the sensitivity of human thyroid caner ARO cells to apoptosis induced by TRAIL by selectively repressing cyclin $\mathrm{D} 1$, a cell cycle regulator commonly overexpressed in thyroid carcinoma (Lloyd et 
al., 1997; Erickson et al., 1998, 2000; Wang et al., 1998). Its overexpression has also been reported to be more frequent in poorly differentiated thyroid tumors. Cyclin D1 repression, in turn, induces G1 cell cycle arrest and reduces the levels of survivin, an anti-apoptotic protein whose expression is cell cycle-regulated (Li et al., 1998). Our results provide a novel mechanism underlying the sensitizing effects of tunicamycin on TRAIL-induced apoptosis by repression of cyclin D1 and subsequent downregulation of survivin.

A

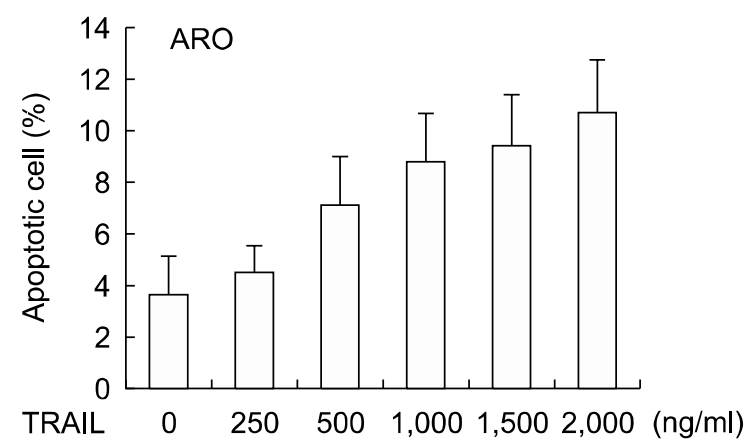

C

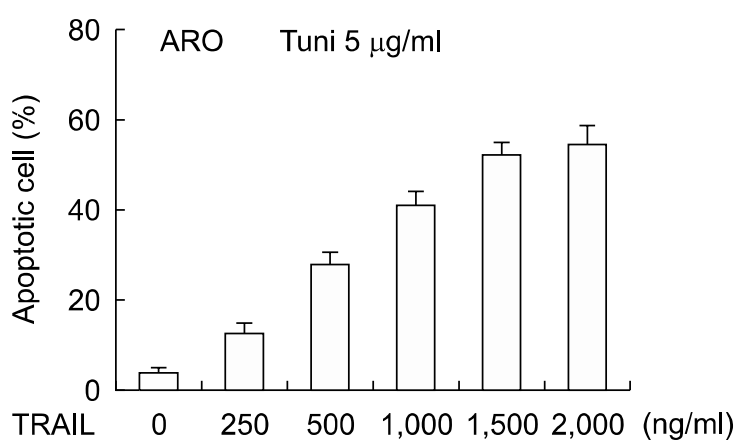

E

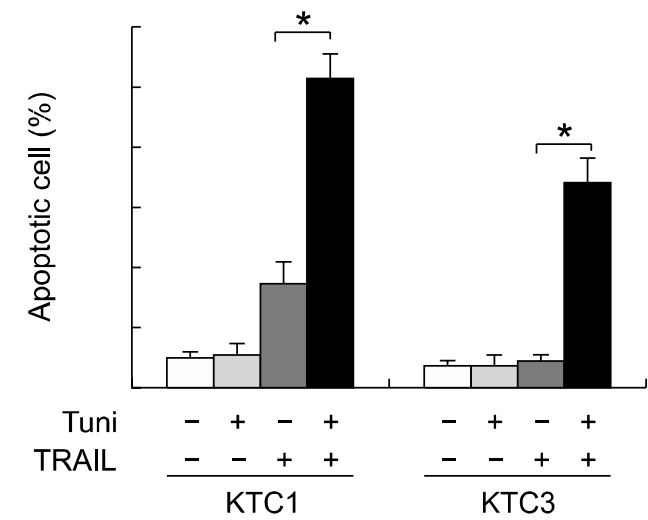

\section{Results}

Subtoxic doses of tunicamycin significantly sensitize human thyroid cancer cells, but not normal human thyroid epithelial cells, to TRAIL-induced apoptosis

The cytotoxic activity of human recombinant soluble TRAIL was tested in ARO undifferentiated thyroid carcinoma cells (Figure 1A). As previously reported, ARO cells demonstrated a limited cell death $(<10 \%)$ upon treatment with up to $2 \mu \mathrm{g} / \mathrm{ml}$ TRAIL
B

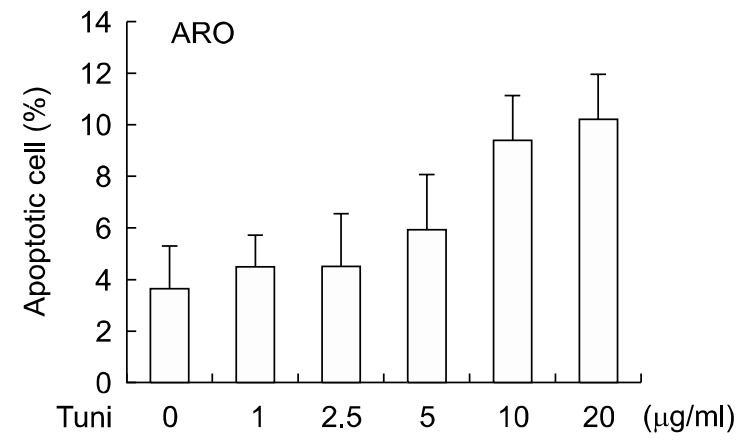

D

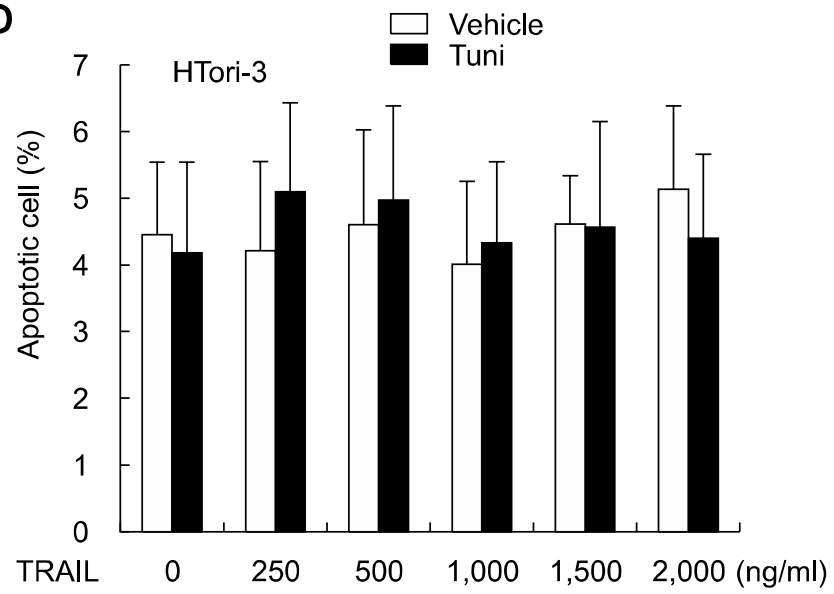

Figure 1. Sensitization of ARO cells by tunicamycin to TRAIL. ARO cells were treated with TRAIL $(A)$ or tunicamycin $(B)$ at the indicated concentrations for $48 \mathrm{~h}$ and apoptosis was assessed. ARO (C) and normal thyroid cells (D) were pretreated with $5 \mu \mathrm{g} / \mathrm{ml}$ of tunicamycin $(\mathrm{Tm})$ for $24 \mathrm{~h}$, and then treated with a serial dose of recombinant TRAIL for additional $24 \mathrm{~h}$. (E) KTC1 and KTC3 cells were treated with tunicamycin $(5 \mu \mathrm{g} / \mathrm{ml})$ and/or TRAIL $(1,000 \mathrm{ng} / \mathrm{ml})$, and cell death was measured. ${ }^{*} P<0.01$. 
for $48 \mathrm{~h}$, suggesting that these cells are resistant to the apoptotic effects of TRAIL. To examine whether tunicamycin might restore the sensitivity of TRAILresistant ARO cells to TRAIL-induced apoptosis, we tested the cytotoxic effect of tunicamycin alone or in combination with TRAIL. Tunicamycin alone did not induce any morphological signs of cell death up to $20 \mu \mathrm{g} / \mathrm{ml}$ (Figure 1B). However, pretreatment with $5 \mu \mathrm{g} / \mathrm{ml}$ tunicamycin for $24 \mathrm{~h}$ then stimulation with TARIL $(0-2 \mu \mathrm{g} / \mathrm{ml})$ for an additional $24 \mathrm{~h}$ dramatically induced cell death, with $>50 \%$ apoptosis induction in cells treated with $5 \mu \mathrm{g} / \mathrm{ml}$ tunicamycin and $1.5 \mu \mathrm{g} / \mathrm{ml}$ TRAIL (Figure 1C).

Next we investigated whether the combined treatment undermines the tumor selectivity of TRAIL. Human differentiated thyroid epithelial HTori-3 cells were resistant to TRAIL and tunicamycin, moreover, their viability was not significantly affected by the combined treatment with tunicamycin and TRAIL for $24 \mathrm{~h}$ (Figure 1D). These results suggest that sensitizing regimens using tunicamycin with TRAIL may be preferentially toxic for undifferentiated thyroid carcinoma cells over normal thyroid epithelial cells.

Tunicamycin also sensitized otherwise insensitive thyroid cancer KTC1 and KTC3 cells (Figure 1E), suggesting that it might generally enhance the antitumoral effects of TRAIL in thyroid cancer cells.

\section{Tunicamycin reduces expression of survivin in thyroid cancer cells}

A previous report showed that tunicamycin increased the sensitivity of prostate cancer cells to TRAIL via induction of $\mathrm{CHOP}$ and subsequent upregulation of DR5 (Shiraishi et al., 2005). In tunicamycintreated ARO cells, only minor induction of $\mathrm{CHOP}$ was demonstrated, furthermore, no induction of DR5 was observed (Figure 2A). This suggested that other unidentified molecules might mediate the promoting effect of tunicamycin in ARO cells. To explore the underlying mechanisms by which tunicamycin sensitizes TRAIL-induced apoptosis in ARO cells, we analyzed the expression of several apoptosis-related proteins following treatment with tunicamycin and TRAIL. Treatment with tunicamycin alone led to a significant decrease in survivin and clAP-2 protein levels, whereas XIAP, cIAP-1, $\mathrm{Bcl}-2$ and $\mathrm{Bcl}-\mathrm{xL}$ remained unaltered (Figure $2 \mathrm{~B}$ ). The combined treatment did neither change the expression levels of DR4, DcR1, DcR2 nor FLICEinhibitory protein (FLIP) (Figure 2B).

\section{Overexpression of survivin compromises the sensitizing effect of tunicamycin on TRAIL in thyroid cancer cells}

To examine whether this downregulation of survivin by tunicamycin is critical to trigger TRAIL-induced apoptosis, we established stable cell lines over-

\section{A}

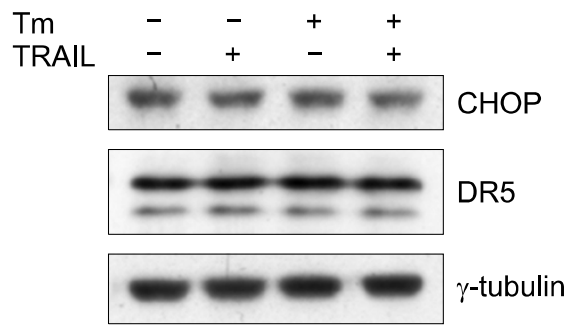

B

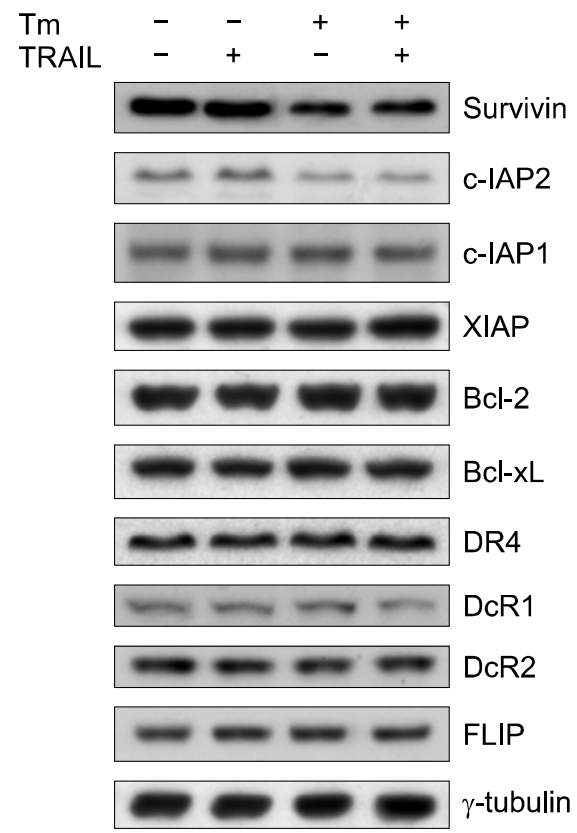

Figure 2. Tunicamycin enhances TRAIL-induced apoptosis by downregulation of survivin. (A) ARO cells were treated with tunicamycin and/or TRAIL for $24 \mathrm{~h}$, and CHOP and DR5 expression was determined by immunoblotting. (B) ARO cells were treated as $(A)$ and western blotting was performed as antibodies indicated. 
A

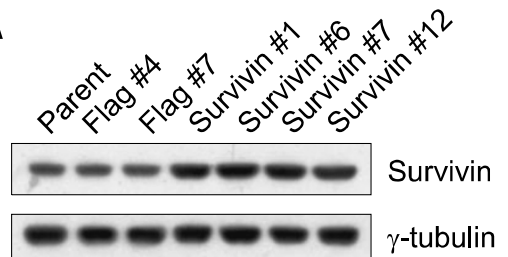

B
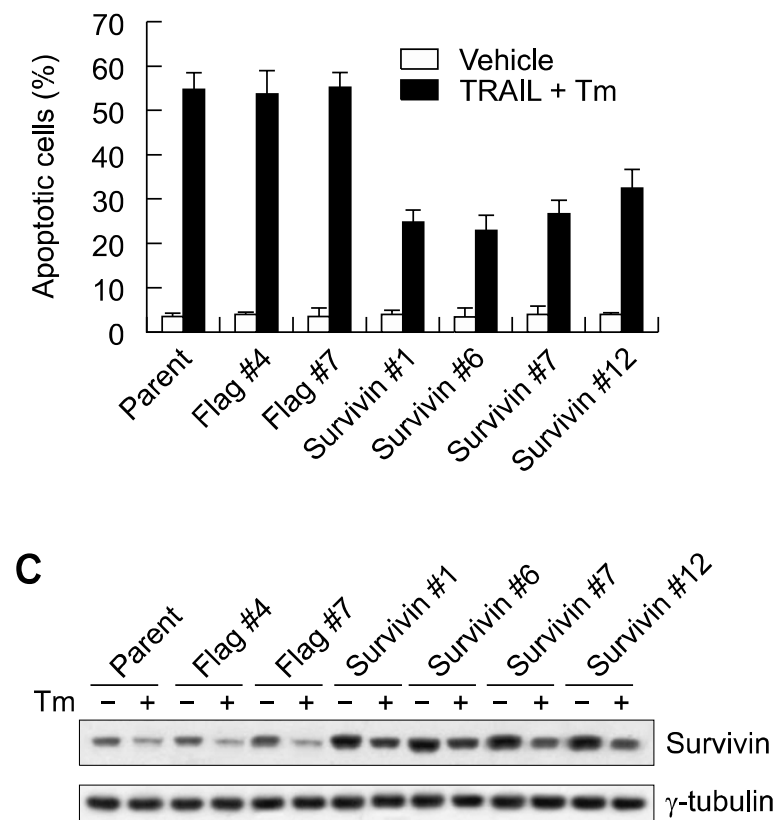

Figure 3. Forced expression of survivin reduces the sensitizing effect of tunicamycin on TRAIL. (A) ARO thyroid cancer cells were stably transfected with plasmids containing empty vector or survivin. (B) ARO cells were pre-treated with tunicamycin for $24 \mathrm{~h}$ and then treated with TRAIL for an additional $24 \mathrm{~h}$, and apoptotic cells were assessed. (C) Parental ARO cells and sublines overexpressing survivin were treated with tunicamycin for $24 \mathrm{~h}$ and protein levels of survivin were evaluated with Western blotting.

expressing survivin (Figure 3A). Ectopic expression of survivin significantly attenuated tunicamycinfacilitated TRAIL-induced apoptosis (Figure 3B). To further confirm whether the difference in survivin expression triggered by tunicamycin accounted for its effect on TRAIL sensitivity, we analyzed the changes in the total protein levels of survivin. The total protein levels of survivin in the tunicamycintreated survivin stable cell lines still remained as high as those in untreated empty vector stable cells, despite the tunicamycin-mediated downregulation of endogenous survivin (Figure $3 \mathrm{C}$ ). Taken together, these results support our hypothesis that downregulation of survivin, at least partially contributes to sensitization of TRAIL.

\section{Tunicamycin reduces survivin levels through downregulation of cyclin D1}

The expression of the IAP family member survivin is cell cycle-dependent with lowest levels at $\mathrm{G} 1$ ( $\mathrm{Li}$ et al., 1998). Tunicamycin has been reported to block G1/S cell cycle progression (Brewer et al., 1999; Hoozemans et al., 2006), and previous studies suggested that agents that can arrest cells in the $\mathrm{G} 1$ phase of the cell cycle may increase TRAIL-induced cytotoxicity (Kim et al., 2005a, b; Lu et al., 2005). We therefore postulated that the reduction in survivin levels secondary to the tunicamycin-induced G1 arrest might account for the TRAIL-sensitizing effects of these drugs. In deed, tunicamycin treatment of ARO thyroid cancer cells potently reduced cyclin D1 protein levels (Figure 4A). Consistent with the established function of cyclin $D$ in promoting cell cycle progression through $\mathrm{G} 1$ to $\mathrm{S}$ phase, the reduction in cyclin D1 levels by tunicamycin led to a dramatic reduction in phosphorylated $\mathrm{Rb}$ (Figure $4 \mathrm{~A}$ ). Cell cycle distribution confirmed that tunicamycin dramatically increased G0/G1 cells (Figure 4B). To examine the specific contribution of cyclin D1 to the decreased survivin level and TRAIL- sensitizing actions of tunicamycin, we selectively suppressed the expression of cyclin D1 by siRNA (Figure 4C). Silencing cyclin D1 inhibited survivin expression to a comparable degree as tunicamycin treatment (Figure 4C). In addition, knockdown of cyclin D1 significantly enhanced TRAIL-induced apoptosis (Figure 4D). To confirm the involvement of survivin and cyclin D1 in sensitizing effect of tunicamycin, we investigated their expression in HTori-3 cells. No survivin was observed and only little cyclin D1 was expressed in HTori-3 (Figure 4E). Collectively, these results suggested that the sensitizing effects of tunicamycin on TRAIL-induced apoptosis mediated at least in part by cyclin D1 downregulation and subsequent suppression of survivin expression.

\section{Ectopic expression of cyclin D1 attenuates the sensitizing effects of tunicamycin on TRAIL-induced apoptosis by preventing downregulation of survivin}

Next, we examined whether forced expression of cyclin D1 could recover tunicamycin-mediated downregulation of survivin. ARO cells were stably transfected with expression vectors encoding cyclin D1 (Figure 5A). Tunicamycin-induced downregulation of survivin was alleviated by overexpression of cyclin D1 (Figure 5B), suggesting that cyclin D1 may be important for regulating survivin expression in these cells. We further investigated 
A

C

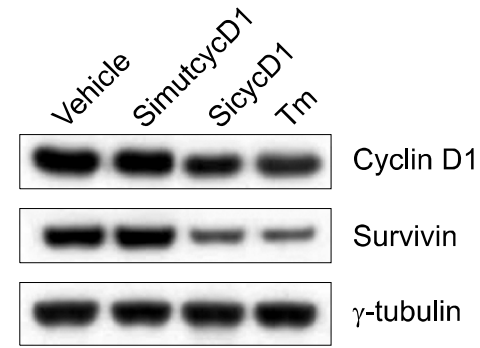

E

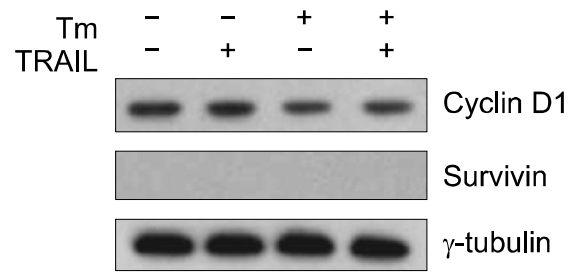

B

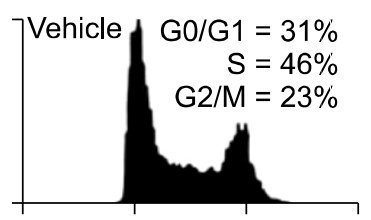

$4 \mathrm{~N}$
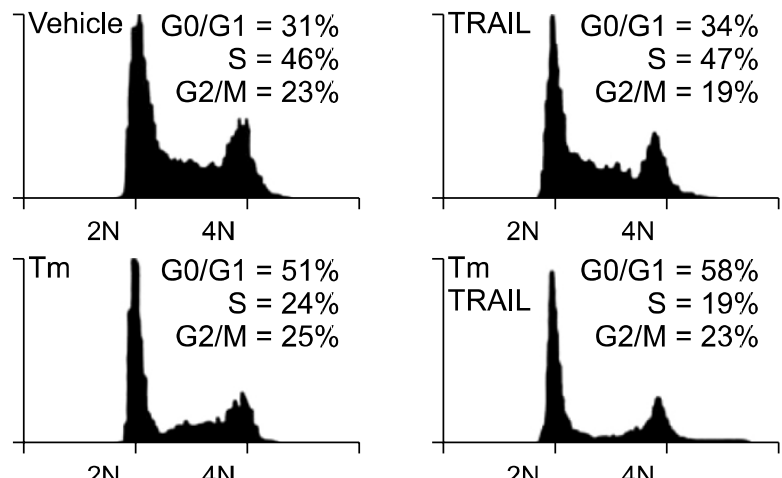

$4 \mathrm{~N}$

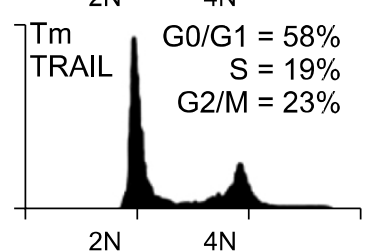

D

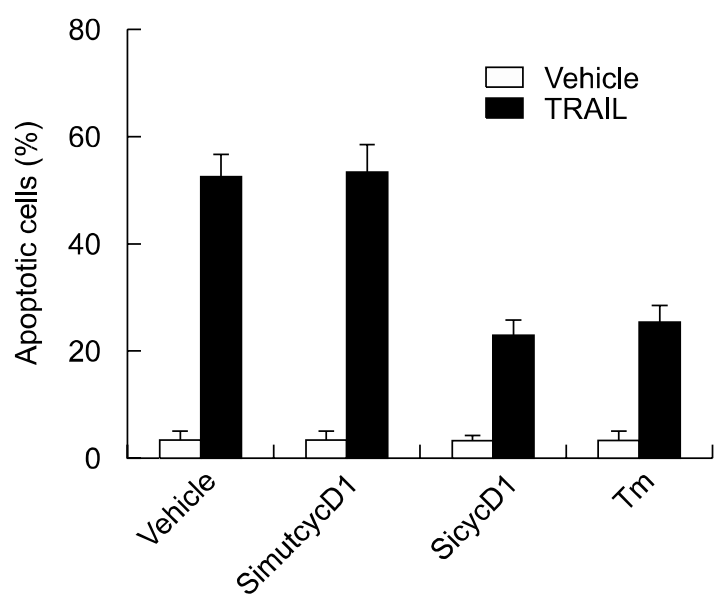

Figure 4. Tunicamycin reduces survivin levels through downregulation of cyclin D1. (A) ARO cells were treated with tunicamycin and/or TRAIL for $24 \mathrm{~h}$, and cyclin D1 and phosphorylated Rb expression was determined by immunoblotting. (B) ARO cells were treated as in (A) and fixed for flow cytometric analysis of DNA content. G0/G1 phase is indicated by the $2 \mathrm{~N}$ peak, $\mathrm{S}$ phase is indicated by the cross-hatched peak, and G2/M phase are indicated by the $4 \mathrm{~N}$ peak. (C) ARO cells were transfected with indicated siRNAs or treated with tunicamycin for $24 \mathrm{~h}$, and protein levels of survivin were determined. (D) ARO cells were treated as in (C), and then treated with TRAIL for additional $24 \mathrm{~h}$, and apoptosis was assessed. (E) HTori-3 cells were treated as in (A) and western blot analysis was performed using the indicated antibodies.

whether forced enhancement of cyclin D1 could over-ride the sensitizing effects of tunicamycin on TRAIL-induced apoptosis. Apoptosis induced by combined treatment with tunicamycin and TRAIL was significantly attenuated by forced expression of cyclin D1 (Figure 5C). Taken together, our results demonstrate that subsequent downregulation of survivin by repression of cyclin D1 may provide one mechanism by which tunicamycin overcomes barriers blocking TRAIL-induced apoptosis of ARO cells.

\section{Discussion}

The efficiency of TRAIL-induced apoptosis in malignant thyroid cancers is variable and many human tumors are resistant to TRAIL-induced apoptosis or acquired resistance to TRAIL during treatment, thereby potentially limiting its therapeutic utility. Hence, it is imperative to identify drugs or agents, which overcome the de novo or acquired resistance of neoplasms to TRAIL-induced apoptosis. Tunicamycin has been used to sensitize spontaneously resistant prostate cancer cells and acquired resistant colon cancer cells to TRAIL-induced apoptosis (Shiraishi et al., 2005). However, the mechanism responsible for the promoting effect of tunicamycin on TRAIL-induced apoptosis is not completely identified. Although previous studies suggested that tunicamycin sensitize prostate cancer cells to TRAIL-induced apoptosis via upregulation of CHOP and subsequent DR5, no increase of DR5 was observed in tunicamycin-treated ARO cells. Here we showed that TRAIL-resistant ARO cells could be converted 
A
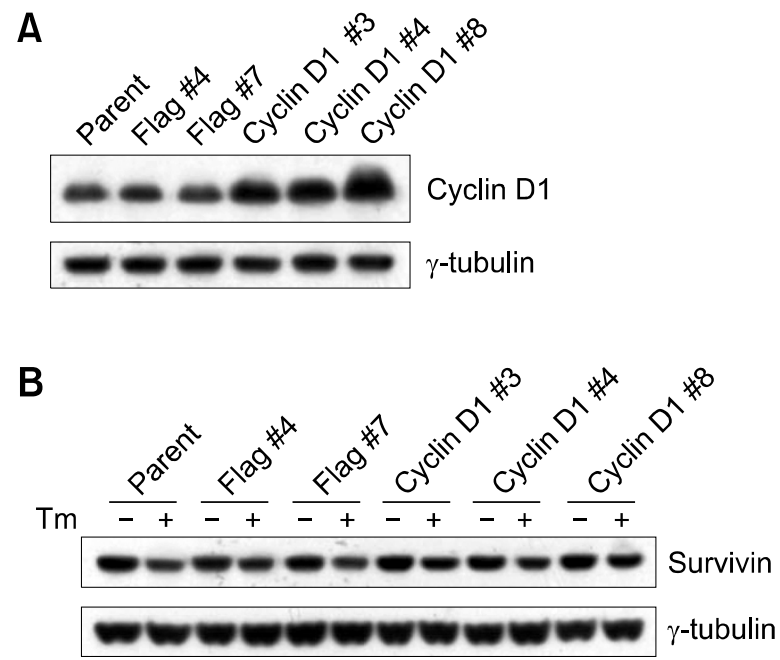

C

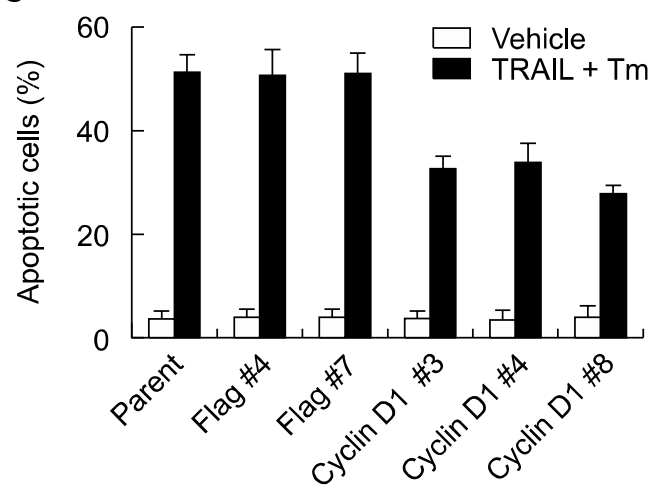

Figure 5. Forced expression of cyclin D1 suppressed the sensitizing effect of tunicamycin on TRAlL by downregulation of survivin. (A) ARO cells were stably transfected with plasmids containing empty vector or cyclin D1. (B) Parental cells and sublines overexpressing cyclin D1 were treated with tunicamycin for $24 \mathrm{~h}$ and protein levels of survivin were evaluated with Western blotting. (C) Cells were pre-treated with tunicamycin for $24 \mathrm{~h}$ and then treated with TRAIL for an additional $24 \mathrm{~h}$, and apoptotic cells were assessed.

to TRAIL-sensitive cells by cotreatment with tunicamycin by suppression of cyclin D1 and subsequent downregulation of survivin.

Survivin is a member of the IAP family that is selectively expressed in many human neoplasms, but not normal adult tissues, and plays a dual role in apoptosis suppression and cell cycle regulation. Previous reports have indicated that survivin is overexpressed in thyroid carcinomas and its high expression level correlated with unfavorable prognosis and resistant to therapy. Indeed, survivin overexpression has been shown to confer protection against many apoptotic stimuli, while inhibition of survivin by a variety of strategies sensitizes cancer cells to apoptosis (Li et al., 1998, 1999; Griffith et al., 2002; Chawla-Sarkar et al., 2004;
Fulda et al., 2004). Since survivin block apoptosis at the effector phase, a point where multiple signaling pathways converge, strategies for removing the inhibitory effects of survivin seem to be potentially useful for overcoming the resistance of cancer cells to anticancer drugs. We found that in parallel with the downregulation of cyclin D1 following treatment with tunicamycin, survivin was dramatically reduced. The functional significance of downregulation of cyclin D1 and the subsequent suppression of survivin expression in tunicamycinenhanced TRAIL-induced apoptosis was confirmed by our observations that silencing cyclin D1 downregulates survivin and promotes TRAIL-induced apoptosis, on the contrary, forced overexpression of cyclin D1 could attenuate tunicamycin-potentiated TRAIL-induced apoptosis, over-riding tunicamycinmediated downregulation of survivin. Furthermore, we have shown that ectopic expression of survivin partially inhibits apoptosis induced by tunicamycin and TRAIL. Thereby, survivin may be a key mediator of TRAIL resistance in thyroid caner cells and that the sensitizing effect of tunicamycin on TRAIL-induced apoptosis may at least partially, result from the downregulation of survivin protein levels via tunicamycin-mediated suppression of cyclin D1, although we cannot exclude the possibility that tunicamycin may have a direct effect on survivin expression as well. Because both cyclin D1 and survivin are amplified and/or overexpressed in a number of human thyroid carcinomas, these tumors may be particularly vulnerable to the combination of tunicamycin and TRAIL.

In our study, combined treatment with tunicamycin and TRAIL was cytotoxic to ARO cells, while differentiated HTori-3 thyroid epithelial cells were spared. Therefore, treatment with TRAIL in combination with subtoxic doses of tunicamycin suggests a potential applicability for treating resistant thyroid cancer cells, although extensive animal studies and preclinical trials using this regimen will be required.

\section{Methods}

\section{Plasmids, antibodies and reagents}

Tunicamycin and recombinant TRAIL were obtained from Sigma (St. Louis, MO) and R\&D systems (Minneapolis, $M N)$, respectively. The following antibodies were used for immunoblotting: DR4, DR5, DcR2 (Stressgen, Victoria, Canada), tubulin (Sigma- Aldrich), DcR1 (BD Biosciences, San Jose, CA), XIAP and survivin (R\&D Systems, Minneapolis, MN), phospho S249 Rb and clAP-2 (Abcam, Cambridge, MA), cyclin D1, clAP-1 (Santa Cruz Biotecnology, Santa Cruz, CA), and FLIP (Novus Biologicals, 
Littleton, CO).

\section{Cell culture}

Human ARO thyroid carcinoma cells and immortalized, differentiated thyroid epithelial HTori-3 cells were obtained from the American Type Culture Collection (ATCC) and European Tissue Culture Collection (ETCC), respectively. KTC1 and KTC3 thyroid carcinoma cells were generously provided by Dr Junichi Kurebayashi (Kawasaki University of Medical Science, Japan). Cells were maintained in RPMI 1640 supplemented with 10\% FBS (Invitrogen, Carlsbad, CA).

\section{Western blot analysis}

Cell lysates were prepared in modified RIPA buffer $(50 \mathrm{mM}$ Tris, $150 \mathrm{mM} \mathrm{NaCl}$ and $1 \%$ Triton X-100) with complete protease inhibitors cocktail. Cell lysate was analyzed by western blot analysis with ECL detection reagents (Amersham Bioscience, Buckinghamshire, UK).

\section{Construction of survivin or cyclin D1 plasmid and generation of ARO cells stably overexpressing survivin or cyclin D1}

A cDNA encoding human survivin or cyclin D1 was generated by PCR from human brain CDNA library (Invitrogen, Carlsbad, CA) and subcloned into the eukaryotic expression plasmid pcDNA3. The constructs were verified by DNA sequencing. Cells were transfected with Lipofectamine 2000 reagent (Invitrogen, Carlsbad, CA) as instructed by the supplier. Control cells were transfected with the pcDNA3-Flag. Transfected cells were selected in medium containing G418 $(800 \mu \mathrm{g} / \mathrm{ml})$ and resistant colonies were expanded. Western analyses employing the antibodies to survivin, cyclin D1 or Flag were used to identify positively expressing cells.

\section{Small interfering RNA (siRNA)}

The siRNA sequences used here were as follows: siRNA against cyclin D1 (sicycD1), GUUCAUUUCCAAUCCGCCC. The scramble nonsense siRNA (scramble; CCGUAUCGUAAGCAGUACU) that has no homology to any known genes was used as control. In addition, position mismatched (sequence underlined) siRNA against cyclin D1 (simutcycD1; GUUCAUUUGGAAUCCGCCC) was also used to confirm the specificity of sicycD1. Transfection of siRNA oligonucleotide was performed with Lipofectamine 2000 (Invitrogen, Carlsbad, CA) according to the manufacturer's recommendations.

\section{Detection of cell death}

Cell death was measured by Annexin-PE Apoptosis Detection Kit I according to the manufacturer's protocol (BD Bioscience), samples were analyzed by fluorescenceactivated cell scanner (FACScan) flow cytometer (Becton Dickinson, Franklin Lakes, NJ). At least 500 nuclei were scored per experiment and all experiments were performed three times.

\section{Flow cytometry}

After fixation with $70 \%$ ethanol, cells were rehydrated in PBS, stained with propidium iodide (Sigma) and analyzed for DNA content by flow cytometry (FACScan, Becton Dickinson). Cell cycle distribution was modeled using ModFit software (Verity Software House).

\section{Data analysis}

Statistical difference was evaluated using the two-tailed Student's $t$ test; $P<0.05$ was considered significant.

\section{Acknowledgements}

We thank Dr Junichi Kurebayashi (Kawasaki University of Medical Science, Japan) for generously providing KTC1 and KTC3 cell lines. This work obtained partial financial support to Wei-Wei Deng from the Shenyang Science and Technology Bureau (No. 1063316-1-00).

\section{References}

Ain KB. Anaplastic thyroid carcinoma: behavior, biology, and therapeutic approaches. Thyroid 1998;8:715-26

Bretz JD, Arscott PL, Myc A, Baker JR, Jr. Inflammatory cytokine regulation of Fas-mediated apoptosis in thyroid follicular cells. J Biol Chem 1999a;274:25433-8

Bretz JD, Rymaszewski M, Arscott PL, Myc A, Ain KB, Thompson NW, Baker JR Jr. TRAIL death pathway expression and induction in thyroid follicular cells. J Biol Chem 1999b;274:23627-32

Brewer JW, Hendershot LM, Sherr CJ, Diehl JA. Mammalian unfolded protein response inhibits cyclin D1 translation and cell-cycle progression. Proc Natl Acad Sci USA 1999;96: 8505-10

Chawla-Sarkar M, Bae SI, Reu FJ, Jacobs BS, Lindner DJ, Borden EC. Downregulation of Bcl-2, FLIP or IAPs (XIAP and survivin) by siRNAs sensitizes resistant melanoma cells to Apo2L/TRAIL-induced apoptosis. Cell Death Differ 2004;11: 915-23

Erickson LA, Jin L, Wollan PC, Thompson GB, van Heerden J, Lloyd RV. Expression of p27kip1 and Ki-67 in benign and malignant thyroid tumors. Mod Pathol 1998;11:169-74

Erickson LA, Yousef OM, Jin L, Lohse CM, Pankratz VS, Lloyd RV. p27kip1 expression distinguishes papillary hyperplasia in Graves' disease from papillary thyroid carcinoma. Mod Pathol 2000;13:1014-9

Fulda S, Debatin KM. Sensitization for tumor necrosis factor-related apoptosis-inducing ligand-induced apoptosis by the chemopreventive agent resveratrol. Cancer Res 2004;64:337-46

Griffith TS, Fialkov JM, Scott DL, Azuhata T, Williams RD, Wall NR, Altieri DC, Sandler AD. Induction and regulation of 
tumor necrosis factor-related apoptosis-inducing ligand/ Apo-2 ligand-mediated apoptosis in renal cell carcinoma. Cancer Res 2002;62:3093-9

Hoozemans JJ, Stieler J, van Haastert ES, Veerhuis R, Rozemuller AJ, Baas F, Eikelenboom P, Arendt T, Scheper $W$. The unfolded protein response affects neuronal cell cycle protein expression: implications for Alzheimer's disease pathogenesis. Exp Gerontol 2006;41:380-6

Kim EH, Kim HS, Kim SU, Noh EJ, Lee JS, Choi KS. Sodium butyrate sensitizes human glioma cells to TRAIL-mediated apoptosis through inhibition of Cdc2 and the subsequent downregulation of survivin and XIAP. Oncogene 2005a;24: 6877-89

Kim EH, Kim SU, Choi KS. Rottlerin sensitizes glioma cells to TRAIL-induced apoptosis by inhibition of Cdc2 and the subsequent downregulation of survivin and XIAP. Oncogene 2005b;24:838-49

LeBlanc HN, Ashkenazi A. Apo2L/TRAIL and its death and decoy receptors. Cell Death Differ 2003;10:66-75

Li F, Ambrosini G, Chu EY, Plescia J, Tognin S, Marchisio PC, Altieri DC. Control of apoptosis and mitotic spindle checkpoint by survivin. Nature 1998;396:580-4

Li F, Ackermann EJ, Bennett CF, Rothermel AL, Plescia J, Tognin S, Villa A, Marchisio PC, Altieri DC. Pleiotropic cell-division defects and apoptosis induced by interference with survivin function. Nat Cell Biol 1999;1:461-6

Lloyd RV, Jin L, Qian X, Kulig E. Aberrant p27kip1 expression in endocrine and other tumors. Am J Pathol 1997;150:401-7

Lu M, Kwan T, Yu C, Chen F, Freedman B, Schafer JM, Lee EJ, Jameson JL, Jordan VC, Cryns VL. Peroxisome proliferator-activated receptor gamma agonists promote TRAIL-induced apoptosis by reducing survivin levels via cyclin D3 repression and cell cycle arrest. J Biol Chem

\section{$2005 ; 280: 6742-51$}

Mitsiades N, Poulaki V, Tseleni-Balafouta S, Koutras DA, Stamenkovic I. Thyroid carcinoma cells are resistant to FAS-mediated apoptosis but sensitive to tumor necrosis factor-related apoptosis-inducing ligand. Cancer Res 2000; 60:4122-9

Pan G, Ni J, Wei YF, Yu G, Gentz R, Dixit VM. An antagonist decoy receptor and a death domain-containing receptor for TRAIL. Science 1997a;277:815-8

Pan G, O'Rourke K, Chinnaiyan AM, Gentz R, Ebner R, Ni J, Dixit VM. The receptor for the cytotoxic ligand TRAIL. Science 1997b;276:111-3

Shiraishi T, Yoshida T, Nakata S, Horinaka M, Wakada M, Mizutani Y, Miki T, Sakai T. Tunicamycin enhances tumor necrosis factor-related apoptosis-inducing ligand-induced apoptosis in human prostate cancer cells. Cancer Res 2005;65:6364-70

Sprick MR, Weigand MA, Rieser E, Rauch CT, Juo P, Blenis J, Krammer PH, Walczak H. FADD/MORT1 and caspase-8 are recruited to TRAIL receptors 1 and 2 and are essential for apoptosis mediated by TRAIL receptor 2 . Immunity 2000;12:599-609

Wang J, Chun HJ, Wong W, Spencer DM, Lenardo MJ. Caspase-10 is an initiator caspase in death receptor signaling. Proc Natl Acad Sci USA 2001;98:13884-8

Wang S, Wuu J, Savas L, Patwardhan N, Khan A. The role of cell cycle regulatory proteins, cyclin D1, cyclin E, and p27 in thyroid carcinogenesis. Hum Pathol 1998;29:1304-9

Wang SH, Mezosi E, Wolf JM, Cao Z, Utsugi S, Gauger PG, Doherty GM, Baker JR, Jr. IFNgamma sensitization to TRAIL-induced apoptosis in human thyroid carcinoma cells by upregulating Bak expression. Oncogene 2004;23:928-35 